Article

\title{
Rcs Phosphorelay Responses to Truncated Lipopolysaccharide-Induced Cell Envelope Stress in Yersinia enterocolitica
}

\author{
Jiao Meng ${ }^{\dagger}$, Junhong $\mathrm{Xu}^{\dagger}$, Can Huang and Jingyu Chen * (1) \\ Beijing Laboratory for Food Quality and Safety, College of Food Science \& Nutritional Engineering, \\ China Agricultural University, Beijing 100083, China; jiaomeng123@yeah.net (J.M.); \\ xujunhong@cau.edu.cn (J.X.); canhuang_cau@hotmail.com (C.H.) \\ * Correspondence: chenjy@cau.edu.cn; Tel.: +86-10-62737966 \\ + These authors contributed equally to this work.
}

Received: 10 November 2020; Accepted: 30 November 2020; Published: 3 December 2020

check for updates

\begin{abstract}
Lipopolysaccharide (LPS) is the major component of the outer membrane of Gram-negative bacteria, and its integrity is monitored by various stress response systems. Although the Rcs system is involved in the envelope stress response and regulates genes controlling numerous bacterial cell functions of Yersinia enterocolitica, whether it can sense the truncated LPS in Y. enterocolitica remains unclear. In this study, the deletion of the Y. enterocolitica waaF gene truncated the structure of LPS and produced a deep rough LPS. The truncated LPS increased the cell surface hydrophobicity and outer membrane permeability, generating cell envelope stress. The truncated LPS also directly exposed the smooth outer membrane to the external environment and attenuated the resistance to adverse conditions, such as impaired survival under polymyxin B and sodium dodecyl sulfate (SDS) exposure. Further phenotypic experiment and gene expression analysis indicated that the truncated LPS was correlated with the activation of the Rcs phosphorelay, thereby repressing cell motility and biofilm formation. Our findings highlight the importance of LPS integrity in maintaining membrane function and broaden the understanding of Rcs phosphorelay signaling in response to cell envelope stress, thus opening new avenues to develop effective antimicrobial agents for combating Y. enterocolitica infections.
\end{abstract}

Keywords: Yersinia enterocolitica; lipopolysaccharide; envelope stress; Rcs phosphorelay

\section{Introduction}

Yersinia enterocolitica is primarily a zoonotic pathogen frequently associated with human non-specific gastroenteritis [1-3]. Y. enterocolitica is listed in the annual reports of the European Food Safety Authority as the third most common enteropathogen, after Campylobacter and Salmonella [4]. In some countries, it is on par with Salmonella as a foodborne pathogen [5]. Y. enterocolitica can flourish at refrigeration temperatures and survive in different harsh environmental conditions, rendering it an important pathogen associated with foodborne infections [6]. Y. enterocolitica is widely distributed in the environment and can be found in soil, water, animals, and various food products [7]. Y. enterocolitica can invade the intestinal mucosa, multiplying and replicating within a phagocytic vacuole [8]. Y. enterocolitica is exposed to a range of envelope stresses due to both their environment and infectious nature [9]. Encountered stresses include desiccation, changing osmotic stress, temperature fluctuations, and exposure to cationic antimicrobial peptides [8,9]. Therefore, Y. enterocolitica transitions through a variety of environments and must respond to these envelope stresses with appropriate gene expression regulation to promote survival and growth within host tissues $[9,10]$. 
The Rcs (regulator of capsule synthesis) phosphorelay system, a non-orthodox two-component regulatory system found in many members of Enterobacterales, is one of the envelope stress response pathways [11,12]. The Rcs system can sense envelope damage or defects and regulate the transcriptome to relieve stress, which is particularly important for the survival and virulence of pathogenic bacteria [13]. The Rcs system is composed of three core proteins, the transmembrane sensor kinase $\mathrm{RcsC}$, the transmembrane protein $\mathrm{RcsD}$, and the response regulator $\operatorname{RcsB}[8,11,12]$. In addition, an outer membrane-associated lipoprotein RcsF is required for the perception of many of the signals that have been shown to activate the Rcs phosphorelay [14]. In the signal transduction of the Rcs phosphorelay, RcsF senses the envelope stress signals and transmits it to RcsC [15]. With the participation of ATP, the hybrid sensor RcsC autophosphorylates at the conserved histidine residue His479 on its histidine kinase $(\mathrm{HK})$ domain. Then, the phosphoryl group is transferred to the aspartate residue Asp875 on the phosphoryl receiver (PR) domain of RcsC. The phosphoryl group is subsequently transferred to the histidine residue His842 on the histidine-containing phosphotransmitter (HPT) domain of RcsD and finally to the aspartate residue Asp56 on the PR domain of RcsB [11,12]. RcsB can form homodimers or heterodimers with auxiliary proteins, such as RcsA, BglJ, MatA, GadE, and then bind to the conserved motif in Rcs-regulated genes to activate or inhibit their transcription [14,16-18]. In general, the Rcs system is activated by outer membrane damage, lipopolysaccharide (LPS) synthesis defects, peptidoglycan perturbation, and lipoprotein mislocalization, which then regulates genes involved in capsule biosynthesis, motility, biofilm formation, and virulence [12].

LPS is an essential structural molecule in the outermost part of the cell envelope, and it consists of three parts: lipid A, core polysaccharide, and O-specific polysaccharide (O-antigen) [19,20]. LPS is a physical barrier that can protect bacteria from the surrounding environment and is recognized by the immune system as a marker for detecting the invasion of bacterial pathogens responsible for the development of inflammatory reactions, and in extreme cases, it is resistant to internal toxic shock [21]. In addition to the role of the O-antigen in bioactivity, the structural integrity of LPS has important implications for bacterial survival and virulence, especially the relatively structurally conserved core polysaccharide [22,23]. Furthermore, defects in LPS biosynthesis can induce cell envelope stress, activating the Rcs system [24,25]. It has been reported that LPS defects impaired motility by repressing flagella gene expression through the Rcs signaling pathway in Escherichia coli, and only mutants with large LPS truncations showed significant defects in those behaviors [26]. Similarly, in a series of mutant strains of E. coli K12 that knocked out the core polysaccharide transferase gene in the waa gene cluster, Ren and colleagues found that only the $\Delta$ waaF strain might be sensed by the Rcs phosphorelay, leading to the production of colanic acid [23]. Although the Rcs system is involved in envelope stress responses and regulates various physiological behaviors of $Y$. enterocolitica $[8,10]$, whether it can sense the truncated LPS in Y. enterocolitica is still unclear.

In this study, we showed that the deletion of the Y. enterocolitica waaF gene truncated the structure of LPS and generated a deep rough LPS. The truncated LPS increased the cell surface hydrophobicity and outer membrane permeability, generating cell envelope stress. This defect in LPS also caused changes in membrane surface smoothness and increased the sensitivity of Y. enterocolitica to environmental stress. The phenotypic experiment and gene expression analysis indicated that the truncated LPS was correlated with the activation of the Rcs phosphorelay, thereby repressing cell motility and biofilm formation. These findings reveal the importance of LPS integrity in maintaining membrane function and further deepen the understanding of Rcs phosphorelay in response to cell envelope stress in Y. enterocolitica.

\section{Results}

\section{1. waaF Gene Knockout in Y. enterocolitica Constructed a Truncated LPS Structure}

In order to obtain a truncated LPS, the core polysaccharide transferase gene waaF was knocked out in the chromosome of wild-type Y. enterocolitica (biotype 1B and serotype O:8). It has been reported 
that core polysaccharides of LPS are synthesized under the corresponding glycosyltransferases and phosphoribosyltransferases encoded by the waa gene cluster $[27,28]$. As each glycosyltransferase is highly substrate specific and temporally sequential, the absence of the waaF gene fails the synthesis of the encoded heptyl glycosyltransferase II, thus leading to a truncated LPS structure [23]. Theoretically, the mutated LPS caused by waaF deletion has only lipids and a portion of the inner core polysaccharides without the outer core polysaccharides and O-antigen, which is called deep rough LPS [22]. In this study, we found that the colony morphology of the $\Delta$ waaF strain had S-R (smooth to rough) variation caused by the waaF deletion (Figure 1A). This may be due to the absence of the LPS O-antigen structure in the $\triangle w a a F$ mutant strain. Furthermore, silver-stained SDS-PAGE showed that most of the sugar chains were deleted from LPS in the $\triangle w a a F$ mutant strain (Figure 1B), which directly verified that the deletion of waaF led to the formation of a truncated LPS structure in Y. enterocolitica.

(A)

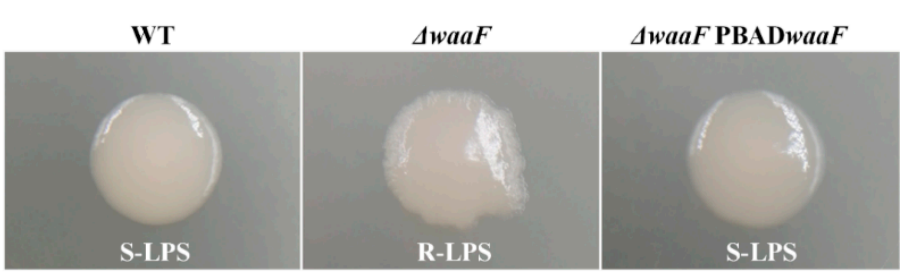

(B)

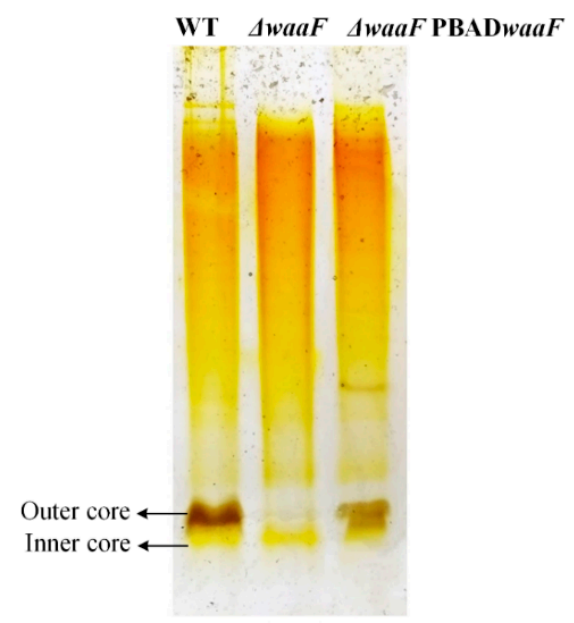

Figure 1. Deep rough lipopolysaccharide (LPS) was constructed by a truncated LPS structure by knocking out the waaF gene in Y. enterocolitica. (A) Colony morphology was transformed from smooth to rough due to the deletion of the waaF gene; (B) A truncated LPS structure was constructed by knocking out the waaF gene through silver-stained SDS-PAGE analysis. Representative images from three independent experiments.

\subsection{Truncated LPS-Induced Envelope Stress in $\triangle$ waaF Strain}

LPS is the central structural and functional unit of the outer membrane of Gram-negative bacteria and plays a crucial role in maintaining the integrity of the cell envelope $[19,20]$. Once its structure is destroyed, the resulting stresses on membranes can be reflected in the structural properties of the bacterial envelope [24,25]. In this study, we measured the permeability and surface hydrophobicity of the outer membrane and observed the cell surface structure by using SEM to measure the changes in membrane properties caused by waaF deletion in Y. enterocolitica. As shown in Figure 2A, the hydrophobicity value of $\Delta w a a F(32.6 \%)$ was significantly higher than that of the wild-type strain $(16.1 \%)$, while the hydrophobicity value of $\triangle$ waaF-PBADwaaF returned to a level similar to that of the wild-type strain. Furthermore, the outer membrane permeability of $\Delta w a a F$ was 1.48 times that of the wild-type strain, but the outer membrane permeability was restored when the mutant was 
complemented with the wild-type waaF gene (Figure 2B). As a result, the cell surface hydrophobicity and outer membrane permeability were increased in the $\Delta$ waa $F$ mutant strain, indicating that the cell envelope stress was induced in response to LPS truncation. In addition, SEM results showed that the outer membrane surface of the $\Delta$ waaF strain had no obvious folds, holes, and other serious membrane damage, while the outer membrane surface of $\Delta$ waaF was relatively smooth when compared to the wild-type strain (Figure 2C).

(A)

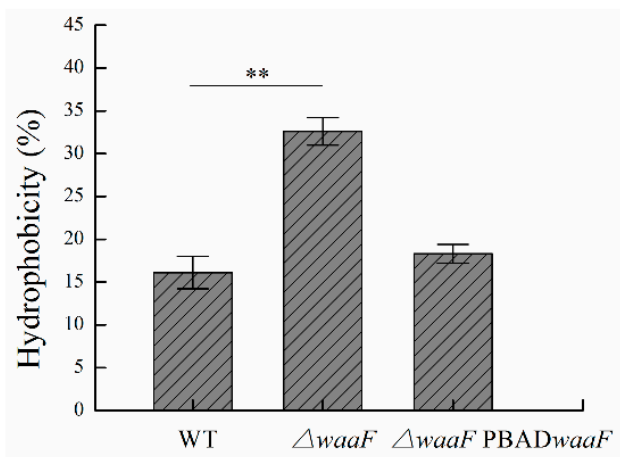

(B)

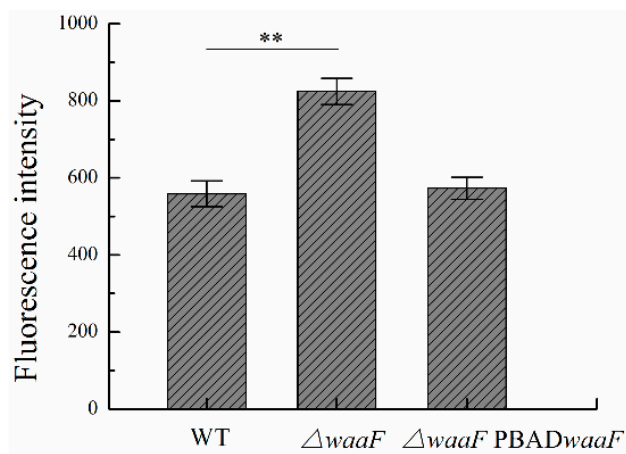

(C)
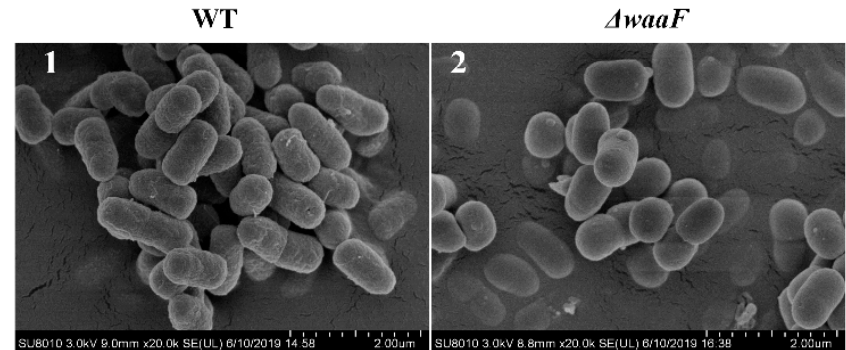

AwaaF PBADwaaF
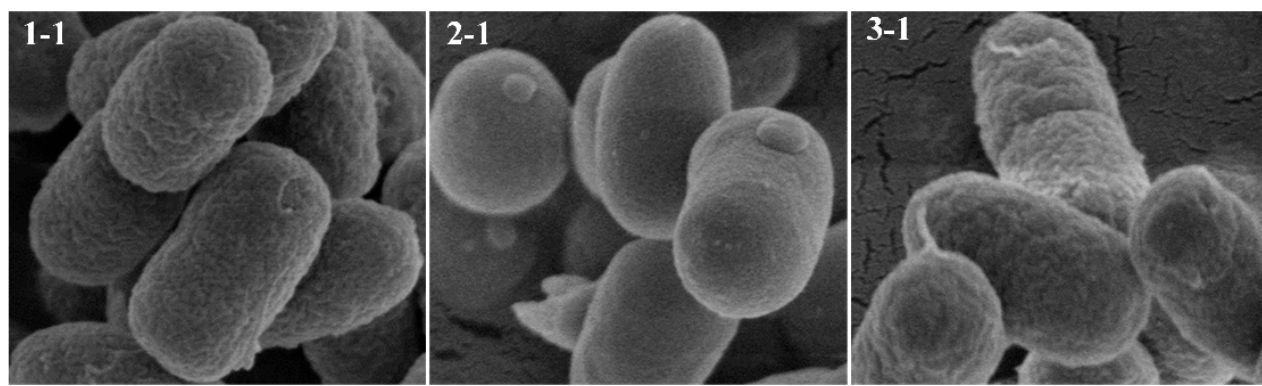

Figure 2. Changes in membrane properties in the wild type, $\Delta w a a F$, and $\Delta w a a F-P B A D w a a F$ strains of Y. enterocolitica. (A) Changes in cell surface hydrophobicity; (B) Changes in outer membrane permeability; (C) Scanning electron microscope images of Y. enterocolitica strains. Among them, sub-figures (1-1, 2-1, 3-1) are partial magnified (100 times) views of sub-figures (1-3), respectively. Data are average values and standard deviations of triplicate experiments. An asterisk indicates a significant difference with ${ }^{* *} p<0.01$.

\subsection{Deletion of waaF Gene Increased Susceptibility of Y. enterocolitica to Environmental Stress}

LPS is a physical barrier for interactions between bacteria and the environment, providing bacteria with great resistance to environmental damage and toxic molecules $[22,29,30]$. To test how defects in LPS structure caused by the lack of waaF affected the resistance to different environmental stresses, the cell growth of wild-type, $\Delta w a a F$, and $\triangle w a a F-P B A D w a a F$ strains exposed to the cationic antimicrobial peptide polymyxin $B$ and anionic surfactant SDS was measured in this study. It should be emphasized here that the lack of waaF does not affect the growth of Y. enterocolitica in the absence of environmental 
stresses (Figure 3A). As shown in Figure 3B, the cell growth of the $\Delta$ waaF strain was significantly inhibited in the presence of $0.4 \mu \mathrm{g}$ polymyxin $\mathrm{B} / \mathrm{mL}$. After $8 \mathrm{~h}$ of incubation, the cell density of the $\Delta w a a F$ strain was decreased by $54 \%$ compared to that of the wild-type strain. However, the growth of the $\Delta w a a F-P B A D w a a F$ strain was close to that of the wild-type strain under polymyxin B stress. Similarly, after the addition of $0.05 \%$ SDS prior to the growth of the bacteria to the logarithmic growth phase, the growth of the $\Delta w a a F$ strain was seriously damaged and slowly recovered in the subsequent cultivation. However, the $\Delta w a a F$ mutant had the ability to grow under $0.05 \%$ SDS stress, but the growth ability was significantly weaker than that of the wild-type strain. The cell density of the $\Delta$ waa $F$ strain decreased by $86 \%$ after $8 \mathrm{~h}$ of incubation compared to that of the wild-type strain. However, the growth of the $\triangle w a a F-P B A D w a a F$ strain with $0.05 \%$ SDS recovered closely to that of the wild-type strain (Figure 3C). These results suggest that LPS truncation caused by waaF deletion increased susceptibility to environmental stress in Y. enterocolitica.

(A)

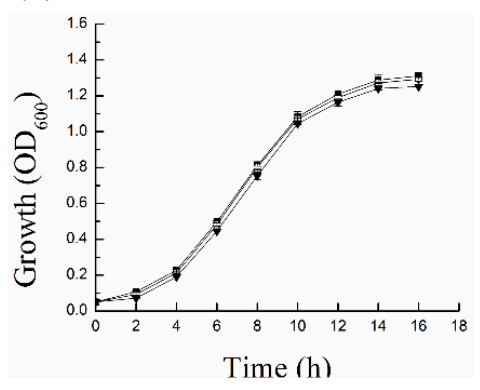

- WT
(B)

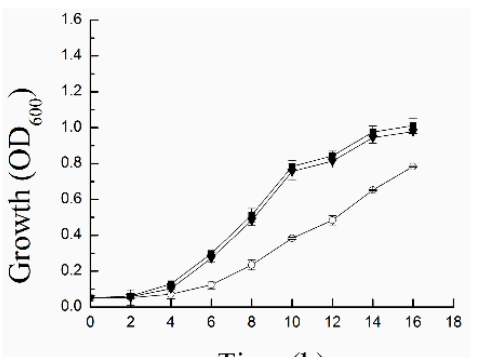

Time (h)
(C)

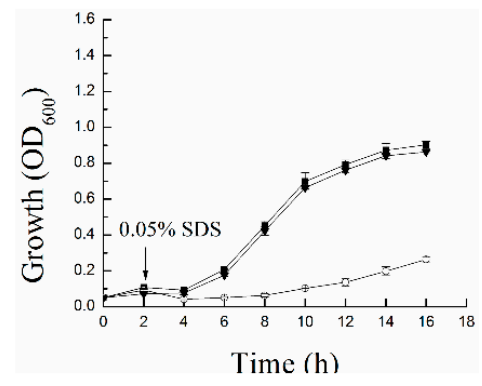

Figure 3. Effect of environmental stresses on the wild type, $\Delta w a a F$, and $\triangle w a a F-P B A D w a a F$ strains of Y. enterocolitica. (A) Growth curves of Y. enterocolitica strains without environmental stresses; (B) Growth curves of $Y$. enterocolitica strains in the presence of $0.4 \mu \mathrm{g}$ polymyxin B/mL; (C) Growth curves of Y. enterocolitica strains in the presence of $0.05 \%$ SDS. The starter cultures were incubated in Luria Broth (LB) medium supplemented with $0.6 \mathrm{~g} / \mathrm{L}$ L-arabinose at $26^{\circ} \mathrm{C}$. Cell growth was measured every $2 \mathrm{~h}$ at $600 \mathrm{~nm}$ in a spectrophotometer. Data are average values and standard deviations of triplicate experiments.

\subsection{Disruption of the Rcs Phosphorelay Reversed the Decreased Motility and Biofilm Formation in $\Delta$ waaF Strain}

In addition to decreased envelope stability and environmental tolerance, the LPS truncation in Enterobacterales also caused abnormal phenotypic changes, including flagella synthesis, biofilm formation, virulence, and pathogenicity [26,31-33]. In this study, swimming motility and biofilm formation were analyzed to investigate the effect of LPS truncation on the phenotypes of Y. enterocolitica. As shown in Figure 4, the $\Delta$ waaF mutant decreased the swim diameter by $53 \%$ in LBNS (LB without salts) agar, but motility was restored when the mutant was complemented with the wild-type waaF gene. Similarly, the defect of LPS in the $\triangle$ waaF strain resulted in a $62 \%$ reduction in biofilm formation, while the $\triangle w a a F-P B A D w a a F$ strain restored biofilm formation after $48 \mathrm{~h}$ of incubation, as evidenced by crystal violet staining when compared to the wild-type strain (Figure 5). These results indicate that LPS truncation caused by waaF mutation decreased motility and biofilm formation in Y. enterocolitica. 


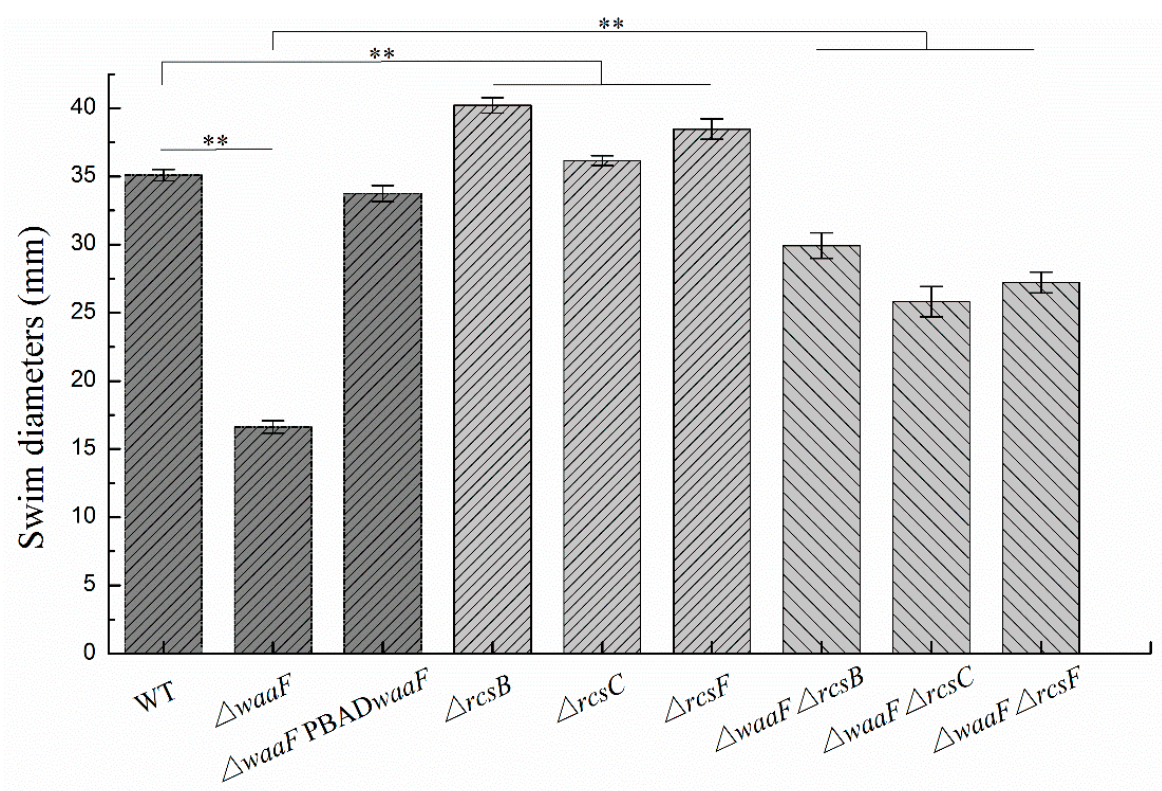

Figure 4. Changes in swim motility of wild-type and mutant strains of Y. enterocolitica. Y. enterocolitica was grown in LBNS semisolid plates supplemented with $0.6 \mathrm{~g} / \mathrm{L} \mathrm{L}$-arabinose at $26{ }^{\circ} \mathrm{C}$. Swim diameters were measured after $48 \mathrm{~h}$ of incubation. Data are average values and standard deviations of triplicate experiments. The statistical comparison shows that there is a significant difference between WT (wild-type) and $\Delta w a a F$; there is a significant difference between WT and $\Delta r c s F / \Delta r c s C / \Delta r c s B$ single mutant; there is a significant difference between $\Delta w a a F$ and $\Delta w a a F-\Delta r c s F / \Delta w a a F-\Delta r c s C / \Delta w a a F-\Delta r c s B$ double mutant. An asterisk indicates a significant difference with ${ }^{* *} p<0.01$.

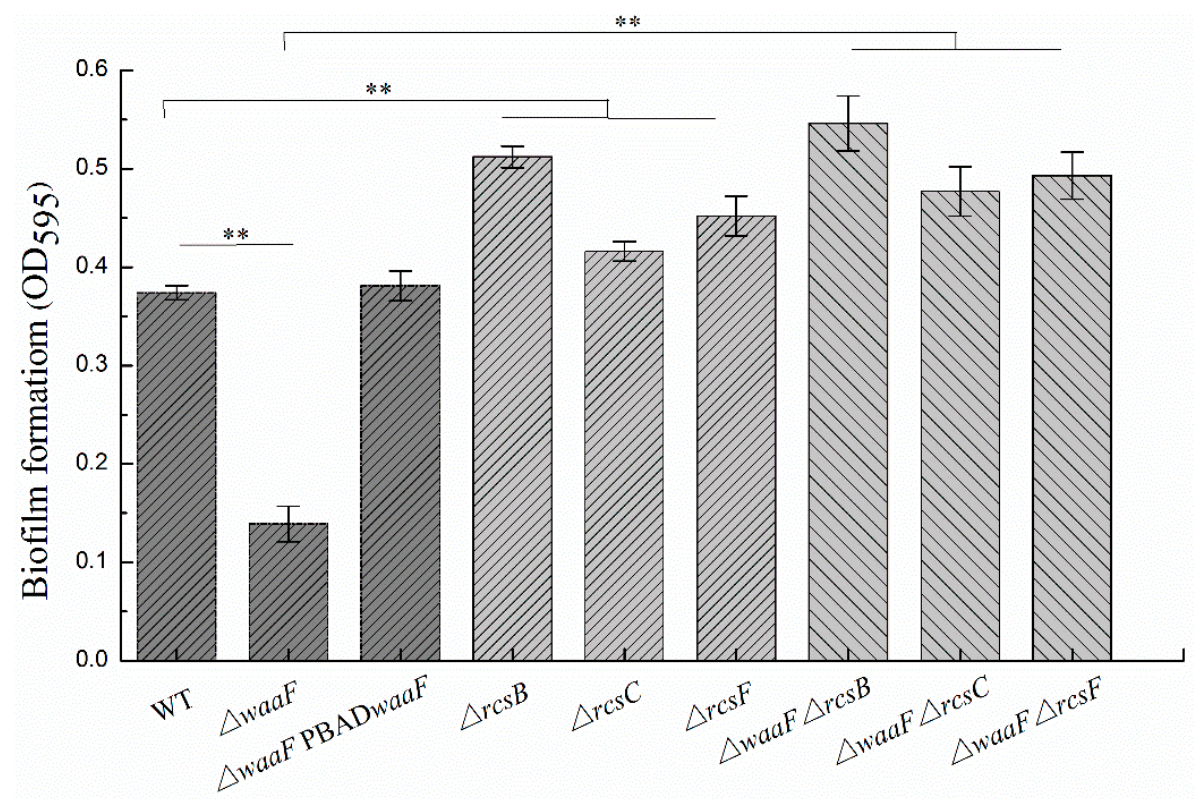

Figure 5. Changes in biofilm formation of wild-type and mutant strains of Y. enterocolitica. Y. enterocolitica was cultured in LNNS (LBNS broth diluted 1:8 in distilled water) medium supplemented with $0.6 \mathrm{~g} / \mathrm{L}$ L-arabinose at $26^{\circ} \mathrm{C}$. Biofilm formation was analyzed after $48 \mathrm{~h}$ of incubation by staining with crystal violet and measuring absorbance at $595 \mathrm{~nm}$. Data are average values and standard deviations of triplicate experiments. The statistical comparison shows that there is a significant difference between WT and $\Delta w a a F$; there is a significant difference between WT and $\Delta r c s F / \Delta r c s C / \Delta r c s B$ single mutant; there is a significant difference between $\Delta w a a F$ and $\Delta w a a F-\Delta r c s F / \Delta w a a F-\Delta r c s C / \Delta w a a F-\Delta r c s B$ double mutant. An asterisk indicates a significant difference with ${ }^{* *} p<0.01$. 
It has been reported that activation of the Rcs phosphorelay triggered by a structural deficiency of LPS suppressed the colanic acid production of $E$. coli, but the effects were reversed after the deletion of any of the Rcs phosphorelay genes [23]. In this study, $\Delta w a a F-\Delta r c s F, \Delta w a a F-\Delta r c s C$, and $\Delta w a a F-\Delta r c s B$ double mutants were constructed to determine whether the disruption of Rcs phosphorelay could reverse the effects caused by waaF deletion in Y. enterocolitica. As a result, the double mutants restored both swim motility and biofilm formation. The $\Delta w a a F-\Delta r c s F, \Delta w a a F-\Delta r c s C$, and $\Delta w a a F-\Delta r c s B$ double mutants increased the swim diameter by 64,55 , and $80 \%$, respectively, compared to the $\Delta w a a F$ strain (Figure 4). Similarly, biofilm formation in $\Delta w a a F-\Delta r c s F, \Delta w a a F-\Delta r c s C$, and $\Delta w a a F-\Delta r c s B$ double mutants showed 2.5-, 2.4-, and 2.9-fold increases, respectively, compared to the $\Delta$ waaF strain after $48 \mathrm{~h}$ of incubation (Figure 5). In addition, the swim diameter and biofilm formation of $\Delta r \operatorname{cs} F, \Delta r c s C$, and $\Delta r c s B$ single mutants were also performed to distinguish the effects of LPS and Rcs phosphorelay on the motility and biofilm formation of $Y$. enterocolitica. The $\Delta r c s F, \Delta r c s C$, and $\Delta r c s B$ single mutants increased the swim diameter by 10,3, and 15\%, respectively, compared to the wild-type strain (Figure 4). The biofilm formation in $\Delta r c s F, \Delta r c s C$, and $\Delta r c s B$ single mutants was increased by 22,14, and $38 \%$, respectively, compared to the wild-type strain (Figure 5). Although the swim diameter and biofilm formation of $\Delta w a a F-\Delta r c s F, \Delta w a a F-\Delta r c s C$, and $\Delta w a a F-\Delta r c s B$ double mutants did not recover to the level of the $\Delta r c s F, \Delta r c s C$, and $\Delta r c s B$ single mutants, it showed that disruption of the Rcs phosphorelay in the $\Delta$ waaF mutant restored motility and biofilm formation to a certain extent in Y. enterocolitica.

\subsection{LPS Truncation Caused by waaF Deletion Activated the Rcs Phosphorelay in Y. enterocolitica}

Our previous data demonstrated that the Rcs phosphorelay represses flhDC, hmsHFRS, and hmsT in Y. enterocolitica [10,34]. The flhDC operon encodes the master regulator of flagella biosynthesis [10]. hmsHFRS and hmsT are genes involved in biofilm formation, of which hmsHFRS required for the biosynthesis of poly- $\beta-1,6-N$-acetylglucosamine exopolysaccharide and $h m s T$ encoding diguanylate cyclase is essential for cyclic dimeric guanosine monophosphate (c-di-GMP) biosynthesis [35]. In this study, we analyzed the transcription levels of these genes to determine whether LPS truncation activated the Rcs phosphorelay in Y. enterocolitica. The results revealed that all these genes were repressed in the $\triangle$ waaF mutant strain. As a result, the transcription of flhDC, hmsHFRS, and hmsT in the $\triangle w a a F$ strain decreased by 49,32 , and $28 \%$, respectively, while transcription was restored in the $\triangle$ waaF-PBADwaaF strain relative to the wild-type strain (Figure 6).

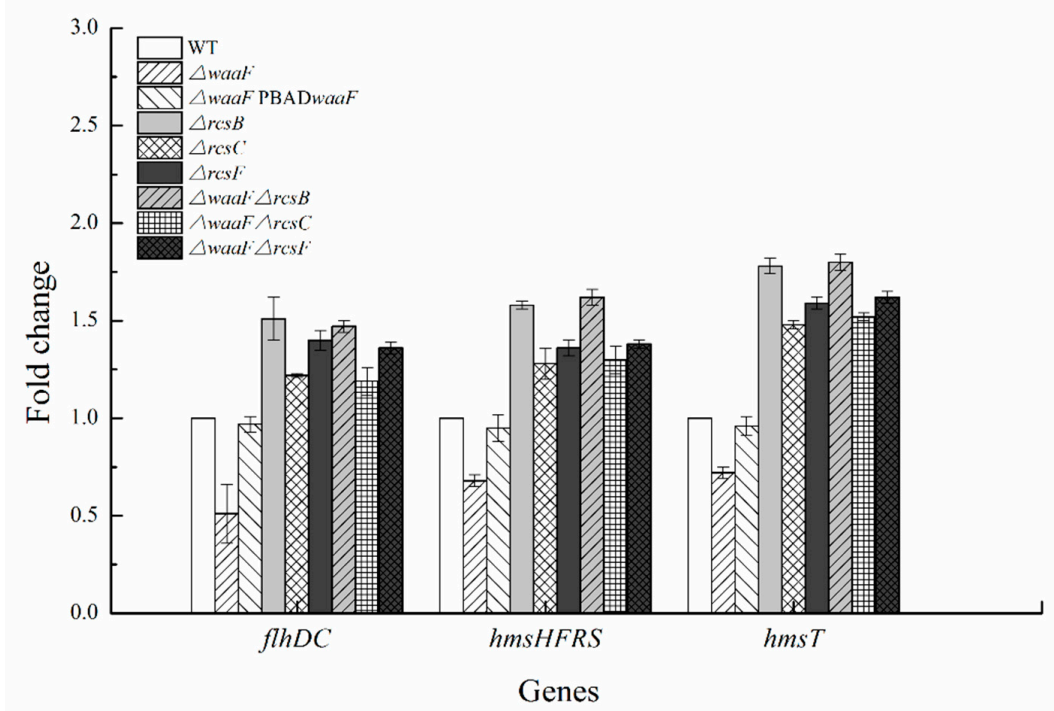

Figure 6. Transcriptional changes in the mutant strains of Y. enterocolitica. Y. enterocolitica was grown to the mid-log phase in LNNS medium, and total RNA was extracted. Expression of $f l h D C, h m s H F R S$, and $h m s T$ was determined by RT-qPCR in vitro. The $16 \mathrm{~S}$ rRNA gene was used as a normalization control. Data are average values and standard deviations of triplicate experiments. 
However, the expression levels of flhDC, hmsHFRS, and $h m s T$ genes downregulated by LPS truncation were increased in all double mutant strains. The transcription of flhDC/hmsHFRS/hmsT in $\Delta w a a F-\Delta r c s F, \quad \Delta w a a F-\Delta r c s C$, and $\Delta w a a F-\Delta r c s B$ was 1.36-times/1.38-times/1.62-times, 1.19-times/1.30-times/1.52-times, and 1.47-times/1.62-times/1.80-times that of the wild-type strain, respectively (Figure 6). The deletion of $r c s F / r c s C / r c s B$ also resulted in an increased expression of these genes. However, there was no significant change between $\Delta$ waaF- $\Delta r c s F, \Delta w a a F-\Delta r c s C$, and $\Delta w a a F-\Delta r c s B$ double mutants and $\Delta r c s F, \Delta r c s C$, and $\triangle r c s B$ single mutants in the transcription of flhDC, hmsHFRS, and hmsT (Figure 6). It can be seen that waaF deletion did not affect the expression levels of these genes in the absence of Rcs phosphorelay in Y. enterocolitica. All these results provide evidence that LPS truncation caused by waaF deletion decreased Y. enterocolitica motility and biofilm formation by acting on the Rcs phosphorelay.

\section{Discussion}

LPS is the major molecular component of the outer membrane of Gram-negative bacteria and serves as a physical barrier providing the bacteria protection from its surroundings $[13,21,29,30]$. Among them, saturated acyl chains and hydrophilic lateral interactions between LPS molecules bridged by divalent cations make the outer membrane impermeable to both large hydrophilic molecules and hydrophobic molecules [13,36]. In this study, the deletion of the waaF gene failed the synthesis of the encoded heptyl glycosyltransferase II in Y. enterocolitica [23], thus leading to a truncated LPS structure (Figure 1B). Theoretically, the mutant LPS has only lipids and a part of the inner polysaccharide, but no outer core and $\mathrm{O}$ antigen [22], so it appears as a deep rough LPS (Figure 1A). The defects in the LPS structure may cause damage to the hydrophilic lateral interaction between LPS and ultimately lead to an increase in membrane permeability in Y. enterocolitica. In addition, the intact LPS showed a gradual increase in hydrophobicity from the inside out in terms of structural composition [20], which may explain why the LPS truncation led to increased hydrophobicity in Y. enterocolitica. It is known that the adhesive capacity closely related to hydrophobicity is important for bacteria to exert their toxic effects [37]. The hydrophobicity of bacteria usually alters when the cell membrane is compromised [37]. In addition to the LPS synthesis defects, some antimicrobial substances that target cell membrane, such as cationic antimicrobial peptide polymyxin B and 3,6-O-[N-(2-aminoethyl)-acetamide-yl]-chitosan (AACS), can also cause changes to the cell surface hydrophobicity $[37,38]$. In general, the truncated LPS structure caused by the deletion of waa $F$ resulted in changes in membrane properties, which eventually led to the generation of envelope stress in Y. enterocolitica.

A new finding of this research is that the deletion of the $Y$. enterocolitica waaF gene renders the outer membrane smoother than the wild-type strain. We thought that the slightly uneven surface of the bacteria observed in the wild type and the $\triangle$ waaF-PBADwaaF strains might be due to the intact structure of LPS. LPS molecules cover more than $70 \%$ of the surface area of the outer membrane of the bacteria [21,22]. One end of its lipid A is anchored to the phospholipids of the outer membrane [39], while the sugar chain composed of core polysaccharides and O-antigen extends freely outside the membrane, endowing the outer membrane of the cell with better ductility so that a rough shape similar to villi is observed through SEM (Figure $2 \mathrm{C}$ ). However, $\triangle$ waaF basically has no polysaccharide chain protrusions extending outside the phospholipids, and the remaining structure of LPS has lost its good ductility. The truncated LPS directly exposed the outer membrane to the external environment without the protection of the intact LPS; therefore, a smooth outer membrane was observed under an SEM (Figure 2C). In addition, changes in the outer membrane permeability in $\Delta$ waaF cells may result in the swelling of the outer membrane during the fixation and drying (sample processing for SEM analysis), thus leading to a smooth outer membrane observed through SEM.

$Y$. enterocolitica is exposed to a range of membrane stresses due to both their environmental and infectious nature, and resistance to such stress is an important property for microbial survival and virulence exertion $[9,10,13]$. The outer membrane of Gram-negative bacteria acts as a protective barrier at the frontline of interaction between bacteria and the environment [13], and the LPS on 
the outer membrane serves as a physical barrier encountered by toxic molecules and antimicrobials, preventing the destruction of cell membranes by these molecules [21,22,29,30]. In agreement with previous reports with $Y$. enterocolitica and similar findings with E. coli, LPS defects led to the decreased resistance to SDS and polymyxin B [22,40]. As we mentioned above, the lateral interaction between LPS prevents macromolecules from penetrating the outer membrane, providing bacteria with great resistance to toxic molecules [13,36]. Furthermore, in Gram-negative bacteria, the known polymyxin $B$ resistance mechanisms involve outer membrane modifications and specifically those in the LPS molecule [41]. In this study, the destruction of the LPS structure and the direct exposure of the outer membrane to the environment due to the lack of protection of the complete LPS structure both can be attributed to the increase in the sensitivity of Y. enterocolitica to polymyxin B and SDS. These results indicate that LPS plays an important role in the survival of Y. enterocolitica in vitro or during the infection of animal tissues.

The Rcs phosphorelay system is an important signal transduction pathway found in many members of the Enterobacterales family [11,12]. This system can integrate environmental signals, regulate gene expression, and alter the physiological behavior of bacteria [42-46]. The outer membrane protein RcsF can sense most of the envelope stress signals that activate the activity of RcsC, trigger the downstream signal transmission of the Rcs system, $\mathrm{RcsC} \rightarrow \mathrm{RcsD} \rightarrow \mathrm{RcsB}$, and finally regulate the transcription of the target gene $[11,12,15]$. It is reported that $E$. coli cells can activate colanic acid production through the Rcs phosphorelay system in response to a truncation of LPS [23]. Konovalova and colleagues proposed a model to explain how RcsF senses LPS defects [24,25]. In their model, RcsF forms a complex with the $\beta$-barrel and uses its positively charged, surface-exposed N-terminal domain to directly sense the state of LPS lateral interactions, thereby regulating the activity of the Rcs system [24,25]. When LPS lateral interactions are perturbed by biosynthesis defect, the RcsF N-terminus will be activated; then, the Rcs system will be triggered [24,25].

In Y. enterocolitica, the Rcs system was reported to sense cell envelope stress and regulate more than 130 genes involved in antibiotic resistance, bacterial chemotaxis, motility, and biofilm formation $[8,10]$. However, whether it can sense LPS truncation has not been reported previously. In this study, we found that the flhDC, hmsHFRS, and hmsT genes regulated by Rcs phosphorelay were repressed by LPS truncation, but the deletion of $r c s F, r c s C$, and $r c s B$, which encode Rcs phosphorelay components in $\triangle w a a F$ cells, restored the expression levels of flhDC, hmsHFRS, and hmsT as well as cell motility and biofilm formation in Y. enterocolitica. In fact, increased $f l h D C, h m s H F R S$, and $h m s T$ were also observed in $\Delta r c s F, \Delta r c s C$, and $\Delta r c s B$ single mutants. However, there was no significant difference between $\Delta w a a F-\Delta r c s F, \Delta w a a F-\Delta r c s C$, and $\Delta w a a F-\Delta r c s B$ double mutants and $\Delta r c s F, \Delta r c s C$, and $\Delta r c s B$ single mutants in terms of the expression level of all these genes, suggesting that LPS truncation could not affect the expression of these genes in the inactivation of Rcs phosphorelay in Y. enterocolitica. A similar effect was also found in the $\triangle o p g G H$ mutant reported by our previous study [10]. The expression of flhDC, hmsT, and hmsHFRS was downregulated by 45, 33, and 34\%, respectively, due to the opgGH (encoding enzymes for synthesizing osmoregulated periplasmic glucans) deletion [10]. In this study, the transcription of $f h D C, h m s T$, and $h m s H F R S$ was decreased by 49,28 , and $32 \%$, respectively, which is caused by waaF deletion. Judging from the expression levels of these Rcs-regulated genes, the lack of waaF and opgGH has little difference in the activation degree of the Rcs system. It should be noted that LPS truncation did not affect the expression of these genes in the absence of RcsF, indicating that the RcsF protein is required for the perception of LPS defects caused by waaF deletion in Y. enterocolitica. All these data provide evidence that the truncated LPS in Y. enterocolitica $\Delta$ waaF cells might be sensed by RcsF and then activate the Rcs system, leading to decreased cell motility and biofilm formation. In addition, Bengoechea and colleagues found that the LPS O-antigen of Y. enterocolitica is required for virulence and the absence of $\mathrm{O}$-antigen also affects the expression of other $Y$. enterocolitica virulence factors [47]; furthermore, the overexpression of O-antigen causes membrane stress that activates the CpxAR two-component signal transduction system [48]. Together, these findings suggest that the 
defects of LPS in the outer membrane of Y. enterocolitica either directly or indirectly, for example through a cellular or envelope stress, could act as a regulatory signal.

\section{Materials and Methods}

\subsection{Bacterial Strains, Plasmids, and Culture Conditions}

The bacterial strains and plasmids used in this study are listed in Table 1. E. coli DH5a, used as the host bacteria in plasmid construction, was cultured at $37^{\circ} \mathrm{C}$ in $\mathrm{LB}$ medium $(5 \mathrm{~g} / \mathrm{L}$ yeast extract, $10 \mathrm{~g} / \mathrm{L}$ tryptone, and $10 \mathrm{~g} / \mathrm{L} \mathrm{NaCl}$ ). Y. enterocolitica ATCC23715 (biotype $1 \mathrm{~B}$ and serotype $\mathrm{O}: 8)$ was used as the parent strain for constructing $Y$. enterocolitica mutants. If not stated otherwise, Y. enterocolitica strains were grown in LB medium or LB agar plates at $26^{\circ} \mathrm{C}$. Ampicillin $(100 \mu \mathrm{g} / \mathrm{mL})$, chloramphenicol $(15 \mu \mathrm{g} / \mathrm{mL}$ ), and CIN (for screening Y. enterocolitica) composed of cefsulodin $(15 \mu \mathrm{g} / \mathrm{mL}$ ), irgasan $(4 \mu \mathrm{g} / \mathrm{mL})$, and novobiocin $(2.5 \mu \mathrm{g} / \mathrm{mL})$ were added as required.

Table 1. Bacterial strains and plasmids used in this study.

\begin{tabular}{|c|c|c|}
\hline Strains and Plasmids & Relevant Characteristics & Sources \\
\hline \multicolumn{3}{|l|}{ Y.enterocolitica } \\
\hline $\mathrm{YE}$ & WT, Serotype O:8, Biotype 1B, pYV- & [34] \\
\hline YE-W & $\Delta w a a F$ & This study \\
\hline YE-W+ & $\Delta w a a F$, PBADwaaF; Ampr & This study \\
\hline YE-F & $\Delta r c s F$ & [10] \\
\hline YE-C & $\Delta r c s C$ & [10] \\
\hline YE-B & $\Delta r c s B$ & [34] \\
\hline YE-WF & $\Delta w a a F, \Delta r c s F$ & This study \\
\hline YE-WC & $\Delta w a a F, \Delta r c s C$ & This study \\
\hline YE-WB & $\Delta w a a F, \Delta r c s B$ & This study \\
\hline \multicolumn{3}{|l|}{ E. coli } \\
\hline S17-1 גpir & $\begin{array}{c}\operatorname{rec} A 1, \text { thi, pro, hsdR-M+, } \\
\text { RP4:2-Tc::Mu-Kan::Tn7, גpir }\end{array}$ & {$[34]$} \\
\hline DH5a & $\begin{array}{c}\text { F-, } \varphi 80 \text { lacZ } \triangle M 15, \Delta(\text { lacZYA-argF }) U 169, \\
\text { deoR, recA1, endA1, hsdR17(rk-, mk+), } \\
\text { pho } A, \sup E 44, \lambda-, \text { thi-1, gyrA96, relA1 }\end{array}$ & [34] \\
\hline \multicolumn{3}{|l|}{ Plasmids } \\
\hline pDS132 & $\begin{array}{c}\text { conditional replication vector; R6K origin, } \\
\text { mobRK4 transfer origin, } \\
\text { sucrose-inducible-sacB; } \mathrm{Cmr}\end{array}$ & [34] \\
\hline pDS132- $\Delta w a a F$ & $\begin{array}{l}\text { upstream and downstream fragments of } \\
\text { waaF gene were cloned into pDS132; Cmr }\end{array}$ & This study \\
\hline $\mathrm{pDS} 132-\Delta r c s F$ & $\begin{array}{l}\text { upstream and downstream fragments of } \\
\text { rcsF gene were cloned into pDS132; Cmr }\end{array}$ & [10] \\
\hline pDS132- $\Delta r c s C$ & $\begin{array}{l}\text { upstream and downstream fragments of } \\
r c s C \text { gene were cloned into pDS132; Cmr }\end{array}$ & [10] \\
\hline $\mathrm{pDS} 132-\Delta r c s B$ & $\begin{array}{l}\text { upstream and downstream fragments of } \\
\text { rcsB gene were cloned into pDS132; Cmr }\end{array}$ & [34] \\
\hline pBAD24 & AraC, promoter PBAD; Ampr & [34] \\
\hline pBAD24-waaF & AraC, PBAD-waaF; Ampr & This study \\
\hline
\end{tabular}

\subsection{Plasmids and Strains Construction}

To construct pDS132- $\Delta w a a F$, fragments upstream and downstream to the waaF gene were amplified from the Y. enterocolitica genome using primers waaF-up-F/waaF-up-R and waaF-down-F/waaF-down- $R$ (Table 2). The upstream and downstream fragments were fused and amplified by fusion PCR with primers waaF-up-F/waaF-down-R (Table 2). The resultant long fragment was digested with $S p h I$ and SacI and ligated into pDS132 digested with the same enzymes to yield pDS132- $\Delta$ waaF. 
Plasmid pDS132- $\Delta$ waaF was introduced into the donor strain E. coli S17-1 $\lambda$ pir by electroporation and then transferred into wild-type Y. enterocolitica ATCC23715 by conjugation. The strategy for constructing the $\triangle w a a F$ mutant was based on the two-step homologous recombination with plasmid pDS132 containing the $s a c B$ counter-selectable marker and a chloramphenicol resistant marker as described previously [10]. Similarly, plasmids pDS132- $\Delta r c s F$, pDS132- $\Delta r c s C$, and pDS132- $\Delta r c s B$ were used to construct the $\Delta w a a F-\Delta r c s F, \Delta w a a F-\Delta r c s C$, and $\Delta w a a F-\Delta r c s B$ double mutants, respectively.

Table 2. Primers used for strain and plasmid construction in this study.

\begin{tabular}{cc}
\hline Primer & Sequences $\left(\mathbf{5}^{\prime} \rightarrow \mathbf{3}^{\prime} \mathbf{)}\right.$ \\
\hline$w a a F-u p-F$ & ATCGCATGCTGCCACAAGCTGATTCACAGA \\
$w a a F-u p-R$ & ACCGTTTATCAATTCCTTGCAGCAAGTTATT \\
$w a a F-$ down $-F$ & AATAACTTGCTGCAAGGAATTGATAAACGGTTGCATGTATTGATCGTTAAAA \\
$w a a F-$ down $-R$ & ATTCAGAGCTCCTGCGCAATAGCATAATCGCC \\
$p-w a a F-F$ & ATGCGTCGACATGAAAATACTGGTAATCG \\
$p-w a a F-R$ & CCCAAGCTTTTAATCGCCCTCTTTCACA \\
$r c s F-u p-F$ & ACTGCATGCAAATCATTGGAAGAACTGCAAC \\
$r c s F-$ down $-R$ & ACTGCGAGCTCCTTTGCGGTAGGCTGGGCGTG \\
$r c s C-u p-F$ & ACTGCATGCCTCAATGGCGACGATCGGGTTA \\
$r c s C-$ down- $R$ & ACTGCGAGCTCCAGATTTAGCCATAATAGTAC \\
$r c s B-u p-F$ & ACTGCATGCAGAAGTGCGTTCTATAATCACA \\
$r c s B-$ down $-R$ & ACTGCGAGCTCATCTGGATGAGAATGCAGATC \\
\hline
\end{tabular}

Restriction sites are underlined.

To construct pBAD24-waaF, the waaF fragment was amplified from the Y. enterocolitica genome using primers $p$-waaF-F/p-waaF-R (Table 2), digested with SalI and HindIII, and inserted into pBAD24 digested with the same enzymes. The pBAD24-waaF plasmid was used to transform the $\Delta$ waa $F$ mutant by electroporation to yield the $\Delta$ waaF-PBADwaaF complemented strain. Ampicillin $(100 \mu \mathrm{g} / \mathrm{mL})$ and L-arabinose $(0.6 \mathrm{~g} / \mathrm{L})$ were added to maintain plasmid pBAD24-waa $F$ and induce waaF expression, respectively, in the $\triangle$ waaF-PBADwaaF strain. All primers used in this study are listed in Table 2.

\subsection{Observation of Colony Morphology}

The wild-type, $\Delta w a a F$ mutant, and $\triangle w a a F-P B A D w a a F$ strains stored at $-80^{\circ} \mathrm{C}$ were streaked on LB agar plates. After the strain was cultured at $26^{\circ} \mathrm{C}$ for $48 \mathrm{~h}$, the morphology of the bacterial colony was observed and photographed with a digital camera.

\subsection{LPS Isolation and Silver Stained SDS-PAGE}

LPS from the wild type, $\triangle w a a F$ mutant, and $\triangle w a a F-P B A D w a a F$ strains were isolated using an LPS extraction kit (iNtRON Biotechnology, Gyeonggi-do, Korea) according to the manufacturer's instructions. The LPS samples were separated by SDS-PAGE using $8-20 \%$ gradient gels (Solarbio, Beijing, China). LPS was stained using the PAGE gel silver staining kit (Solarbio, Beijing, China) according to the manufacturer's instructions. Gels were visualized under the white light of GelDoc-It ${ }^{2}$ (UVP, Upland, CA, USA).

\subsection{Hydrophobicity Test}

Cell surface hydrophobicity was determined by microbial adhesion to hydrocarbons with slight modifications [49]. Hexadecane was chosen as the non-polar solvent to reflect bacterial surface hydrophobicity. Briefly, logarithmic phase bacteria were harvested by centrifugation at $5000 \times g$ for $10 \mathrm{~min}$, washed three times, and resuspended in phosphate buffered solution (PBS) (pH = 7.4), and the bacterial concentration was adjusted to $\mathrm{OD}_{600}=0.5\left(\mathrm{~A}_{0}\right)$. The two-phase system was thoroughly mixed by vortexing for $2 \mathrm{~min}$ after $300 \mu \mathrm{L}$ of hexadecane was added to $1.2 \mathrm{~mL}$ bacterial suspension. The aqueous phase was removed after $30 \mathrm{~min}$ of incubation at $37^{\circ} \mathrm{C}$ to measure the absorbance at $600 \mathrm{~nm}\left(\mathrm{~A}_{1}\right)$. The hydrophobic rate was calculated using the following formula: hydrophobic rate 
$(\%)=\frac{\left(A_{0}-A_{1}\right)}{A_{0}} \times 100$, where $A_{0}$ is the initial absorption value at $600 \mathrm{~nm}$ and $A_{1}$ is the absorption value at $600 \mathrm{~nm}$ of bacteria treated with hexadecane.

\subsection{NPN Uptake Assays}

The outer membrane permeability of $Y$. enterocolitica strains was determined using the $\mathrm{N}$-phenyl-1-naphthylamine (NPN) method, as described previously [37]. NPN is a hydrophobic fluorescent probe that can penetrate into the outer membrane of bacteria. This kind of probe emits a strong fluorescent signal in a hydrophobic environment (i.e., cell envelope), but a weak fluorescent signal in an aqueous environment. Therefore, the fluorescence intensity of NPN can be used to reflect the outer membrane permeability of bacteria. The procedure for NPN determination was as follows: bacteria were harvested in log phase of growth by centrifugation at $5000 \times g$ for $10 \mathrm{~min}$, rinsed three times, and resuspended in PBS buffer $(\mathrm{pH}=7.4)$ to $\mathrm{OD}_{600}=0.5$. An aliquot of $1.98 \mathrm{~mL}$ of bacterial suspension was fully mixed with $20 \mu \mathrm{L}$ NPN $(1 \mathrm{mM})$ and then incubated for $30 \mathrm{~s}$; then, the fluorescence value was measured immediately using a fluorescence spectrophotometer (RF-5301PC, Shimadzu, Japan). Excitation and emission wavelengths for NPN were set at 350 and $429 \mathrm{~nm}$, respectively, with slit widths of $5 \mathrm{~nm}$.

\subsection{Stress Survival Assays}

Growth curves of $Y$. enterocolitica strains with environmental stresses were performed as described previously [22]. Strains were grown overnight at $26{ }^{\circ} \mathrm{C}$ and then diluted in $20 \mathrm{~mL}$ LB medium to an $\mathrm{OD}_{600}$ of $\sim 0.05$. To study the effects of sodium dodecyl sulfate (SDS) on the strains, cells were incubated for $2 \mathrm{~h}$ and $0.05 \%$ SDS was added. For the polymyxin B stress assays, a specific concentration of polymyxin B was added to the LB medium to obtain final concentrations of $0.4 \mu \mathrm{g}$ polymyxin $\mathrm{B} / \mathrm{mL}$. Samples were taken every $2 \mathrm{~h}$ after inoculation, which were stored under $26^{\circ} \mathrm{C}, 180 \mathrm{rpm}$ culture conditions, and $\mathrm{OD}_{600}$ was determined by a spectrometer. Growth curves were plotted according to the determined $\mathrm{OD}_{600}$ and sampling time.

\subsection{Motility Assays}

Swimming motility assays were performed on LBNS plates containing $0.35 \%$ agar as described previously [10]. The overnight activated bacteria were transferred and cultured in the logarithmic phase, and the bacterial concentration was adjusted to $\mathrm{OD}_{600}=1.0$. Then, $1 \mu \mathrm{L}$ bacterial suspensions were inoculated into the center of the swimming plate and incubated at $26^{\circ} \mathrm{C}$. The diameters of the swimming rings were measured using a Vernier caliper after $48 \mathrm{~h}$ of incubation.

\subsection{Biofilm Assays}

Crystalline violet staining was used to measure biofilm formation as described previously [10]. Logarithmic phase bacteria cultured in LNNS liquid medium were added to a 96-well plate so that each well contained $200 \mu \mathrm{L}$ suspensions (initial $\mathrm{OD}_{600}$ about 0.05 ). The suspensions were incubated at $26^{\circ} \mathrm{C}$ for $48 \mathrm{~h}$ and were replaced with fresh medium every $24 \mathrm{~h}$. After the bacterial growth medium was removed, the biofilm was washed twice with PBS and fixed with methanol. Crystal violet staining solution $(0.1 \%)$ and $33 \%$ acetic acid were used to stain the biofilm and release the dye absorbed in the biofilm, respectively, which was then measured at $595 \mathrm{~nm}$.

\subsection{Electron Microscopy of Cell Morphology}

Scanning electron microscopy (SEM) was used to visualize the damage to the outer membrane as described previously [50]. Bacteria grown to the mid-log phase in LB medium were harvested by centrifugation at 5000 $\mathrm{g}$ for $10 \mathrm{~min}$, washed three times, and resuspended in PBS buffer solution $(\mathrm{pH}=7.4)$. The suspension was premixed with an equal volume of $2.5 \%$ glutaraldehyde for $4 \mathrm{~h}$ at $4{ }^{\circ} \mathrm{C}$ and subsequently dehydrated with $25,50,70,80,95$, and $100 \%$ ethanol. The dehydrated samples were 
air-dried immediately, followed by smearing on SEM stubs and gold covering. The micrographs of the envelope were obtained using a SEM (Hitachi SU8010, Tokyo, Japan).

\subsection{RNA Isolation and Quantitative Real-Time PCR (RT-qPCR)}

Total RNA was extracted from the logarithmic phase bacteria in LNNS medium using the TransZol Up Plus RNA Kit (TransGen, Beijing, China). The RT-qPCR kit (TransGen, Beijing, China) used in this experiment reverse-transcribed RNA into cDNA in the presence of random primers, and cDNA amplification was completed in one step in the same reaction system. RT-qPCR was carried out using SYBR Green and the specific primers listed in Table 3 in a Light Cycler 480 II (Roche, Basel, Switzerland) as described previously $[8,10]$. Reactions were performed in triplicate, and the $16 \mathrm{~S}$ rRNA gene was used as a reference for normalization. Relative transcription levels of the target genes were analyzed by the $2^{-\Delta \Delta C t}$ method as described previously [51].

Table 3. Primers used in the RT-qPCR assay.

\begin{tabular}{clc}
\hline Primer & \multicolumn{1}{c}{ Sequences $\left(5^{\prime} \rightarrow \mathbf{3}^{\prime}\right)$} & Amplicon Size $(\mathbf{b p})$ \\
\hline$q-f l h D C-F$ & CCTCAGCGATGTTTCGTCTC & 176 \\
$q-f l h D C-R$ & CTGCAAGTCATCCACACGAG & \\
$q-h m s H F R S-F$ & GATGATGTACCGCCTCCAGA & 96 \\
$q-h m s H F R S-R$ & GTGAATAGTTTCCCGCGCAT & \\
$q-h m s T-F$ & TATAATCGCCGTGGGTTGGA & \multirow{2}{*}{144} \\
$q-h m s T-R$ & CACTAAGGCTTGGTCTCCCA & \\
$16 S r R N A-F$ & GCACGTAATGGTGGGAACTC & 183 \\
$16 S r R N A-R$ & CTCCAATCCGGACTACGACA & \\
\hline
\end{tabular}

\subsection{Statistical Analysis}

All experiments were conducted in triplicate, and the results are expressed as mean \pm SD. One-way analysis of variance was performed in SPSS for Windows 20.0 (SPSS Inc., Chicago, IL, USA).

\section{Conclusions}

This study mainly focused on the effect of LPS integrity in maintaining membrane function and the response regulation of the Rcs phosphorelay system to the truncated LPS in Y. enterocolitica. The deletion of the Y. enterocolitica waaF gene truncated the structure of LPS and produced deep rough LPS. This truncated LPS resulted in changes in cell surface hydrophobicity and outer membrane permeability, which in turn induced the generation of cell envelope stress. LPS truncation also led to a change in the smoothness of the membrane surface and increased susceptibility to environmental stress. The truncated LPS decreased Y. enterocolitica motility and biofilm formation, and this effect was reversed by disruption of the Rcs phosphorelay. Gene expression analysis indicated that Rcs phosphorelay responds to cell envelope stress induced by truncated LPS in Y. enterocolitica. This study reveals the importance of LPS integrity in maintaining membrane function, broadens the understanding of the Rcs phosphorelay system in response to envelope stress, and provides a theoretical basis for the development of bacterial control, prevention, and treatment.

Author Contributions: J.M. and J.X. performed the experiments under the guidance of J.C.; J.C. and J.M. developed the idea for the study and designed the research. J.M., J.X., C.H. and J.C. analyzed the experimental data and drafted the manuscript. All authors have read and agreed to the published version of the manuscript.

Funding: This work was supported by the National Natural Science Foundation of China (31671830) and the Beijing Natural Science Foundation (6202016).

Acknowledgments: The authors would like to thank Dingyu Liu for the substantial contributions to conception, interpretation of data and revised the manuscript.

Conflicts of Interest: The authors declare no conflict of interest. 


\section{References}

1. Huovinen, E.; Sihvonen, L.M.; Virtanen, M.J.; Haukka, K.; Siitonen, A.; Kuusi, M. Symptoms and sources of Yersinia enterocolitica-infection: A case-control study. BMC Infect. Dis. 2010, 10, 122. [CrossRef] [PubMed]

2. Fabrega, A.; Vila, J. Yersinia enterocolitica: Pathogenesis, virulence and antimicrobial resistance. Enferm. Infecc. Microbiol. Clín. 2012, 30, 24-32. [CrossRef] [PubMed]

3. Zadernowska, A.; Chajecka-Wierzchowska, W.; Laniewska-Trokenheim, L. Yersinia enterocolitica: A dangerous, but often ignored, foodborne pathogen. Food Rev. Int. 2014, 30, 53-70. [CrossRef]

4. European Food Safety Authority and European Centre for Disease Prevention and Control. The European Union summary report on trends and sources of zoonoses, zoonotic agents and food-borne outbreaks in 2017. EFSA J. 2018, 16, 5500.

5. Annamalai, T.; Venkitanarayanan, K. Expression of major cold shock proteins and genes by Yersinia enterocolitica in synthetic medium and foods. J. Food Prot. 2005, 68, 2454-2458. [CrossRef]

6. Bari, M.L.; Hossain, M.A.; Isshiki, K.; Ukuku, D. Behavior of Yersinia enterocolitica in foods. J. Pathog. 2011, 2011, 420732. [CrossRef]

7. Rahman, A.; Bonny, T.S.; Stonsaovapak, S.; Ananchaipattana, C. Yersinia enterocolitica: Epidemiological studies and outbreaks. J. Pathog. 2011, 2011, 239391. [CrossRef]

8. Meng, J.; Bai, J.Q.; Chen, J.Y. Transcriptomic analysis reveals the role of RcsB in suppressing bacterial chemotaxis, flagellar assembly and infection in Yersinia enterocolitica. Curr. Genet. 2020, 66, 971-988. [CrossRef]

9. Brzostek, K.; Skorek, K.; Raczkowska, A. OmpR, a central integrator of several cellular responses in Yersinia enterocolitica. Adv. Exp. Med. Biol. 2012, 954, 325-334.

10. Meng, J.; Huang, C.; Huang, X.N.; Liu, D.Y.; Han, B.Z.; Chen, J.Y. Osmoregulated periplasmic glucans transmit external signals through Rcs phosphorelay pathway in Yersinia enterocolitica. Front. Microbiol. 2020, 11, 122. [CrossRef]

11. Guo, X.P.; Sun, Y.C. New insights into the non-orthodox two component Rcs phosphorelay system. Front. Microbiol. 2017, 8, 2014. [CrossRef] [PubMed]

12. Wall, E.; Majdalani, N.; Gottesman, S. The complex Rcs regulatory cascade. Annu. Rev. Microbiol. 2018, 72, 111-139. [CrossRef] [PubMed]

13. Mitchell, A.M.; Silhavy, T.J. Envelope stress responses: Balancing damage repair and toxicity. Nat. Rev. Microbiol. 2019, 17, 417-428. [CrossRef] [PubMed]

14. Castanie-Cornet, M.P.; Cam, K.; Jacq, A. RcsF is an outer membrane lipoprotein involved in the RcsCDB phosphorelay signaling pathway in Escherichia coli. J. Bacteriol. 2006, 188, 4264-4270. [CrossRef]

15. Cho, S.H.; Szewczyk, J.; Pesavento, C.; Zietek, M.; Banzhaf, M.; Roszczenko, P.; Asmar, A.; Laloux, G.; Hov, A.K.; Leverrier, P.; et al. Detecting envelope stress by monitoring $\beta$-barrel assembly. Cell 2014, 159, 1652-1664. [CrossRef]

16. Pristovsek, P.; Sengupta, K.; Lohr, F.; Schafer, B.; von Trebra, M.W.; Ruterjans, H.; Bernhard, F. Structural analysis of the DNA-binding domain of the Erwinia amylovora RcsB protein and its interaction with the RcsAB box. J. Biol. Chem. 2003, 278, 17752-17759. [CrossRef]

17. Venkatesh, G.R.; Kembou Koungni, F.C.; Paukner, A.; Stratmann, T.; Blissenbach, B.; Schnetz, K. BglJ-RcsB Heterodimers Relieve Repression of the Escherichia coli bgl Operon by H-NS. J. Bacteriol. 2010, 192, 6456-6464. [CrossRef]

18. Pannen, D.; Fabisch, M.; Gausling, L.; Schnetz, K. Interaction of the RcsB response regulator with auxiliary transcription regulators in Escherichia coli. J. Biol. Chem. 2016, 291, 2357-2370. [CrossRef]

19. Bialas, N.; Kasperkiewicz, K.; Radziejewska-Lebrecht, J.; Skurnik, M. Bacterial cell surface structures in Yersinia enterocolitica. Arch. Immunol. Ther. Exp. 2012, 60, 199-209. [CrossRef]

20. Wang, X.; Quinn, P.J. Lipopolysaccharide: Biosynthetic pathway and structure modification. Prog. Lipid Res. 2010, 49, 97-107. [CrossRef]

21. Rosenfeld, Y.; Shai, Y. Lipopolysaccharide (Endotoxin)-host defense antibacterial peptides interactions: Role in bacterial resistance and prevention of sepsis. Biochim. Biophys. Acta 2006, 1758, 1513-1522. [CrossRef] [PubMed] 
22. Leskinen, K.; Varjosalo, M.; Li, Z.L.; Li, C.M.; Skurnik, M. Expression of the Yersinia enterocolitica O:3 LPS O-antigen and outer core gene clusters is RfaH-dependent. Microbiology 2015, 161, 1282-1294. [CrossRef] [PubMed]

23. Ren, G.; Wang, Z.; Li, Y.; Hu, X.Q.; Wang, X.Y. Effects of lipopolysaccharide core sugar deficiency on colanic acid biosynthesis in Escherichia coli. J. Bacteriol. 2016, 198, 1576-1584. [CrossRef] [PubMed]

24. Konovalova, A.; Perlman, D.H.; Cowles, C.E.; Silhavy, T.J. Transmembrane domain of surface-exposed outer membrane lipoprotein RcsF is threaded through the lumen of $\beta$-barrel proteins. Proc. Natl. Acad. Sci. USA 2014, 111, E4350-E4358. [CrossRef] [PubMed]

25. Konovalova, A.; Mitchell, A.M.; Silhavy, T.J. A lipoprotein/ $\beta$-barrel complex monitors lipopolysaccharide integrity transducing information across the outer membrane. eLife 2016, 5, e15276. [CrossRef] [PubMed]

26. Girgis, H.S.; Liu, Y.; Ryu, W.S.; Tavazoie, S. A comprehensive genetic characterization of bacterial motility. PLoS Genet. 2007, 3, 1644-1660. [CrossRef]

27. Yethon, J.A.; Heinrichs, D.E.; Monteiro, M.A.; Perry, M.B.; Whitfield, C. Involvement of waaY, waaQ, and waaP in the modification of Escherichia coli lipopolysaccharide and their role in the formation of a stable outer membrane. J. Biol. Chem. 1998, 273, 26310-26316. [CrossRef]

28. Yethon, J.A.; Whitfield, C. Purification and characterization of WaaP from Escherichia coli, a lipopolysaccharide kinase essential for outer membrane stability. J. Biol. Chem. 2001, 276, 5498-5504. [CrossRef]

29. Hancock, R.E.; Diamond, G. The role of cationic antimicrobial peptides in innate host defences. Trends Microbiol. 2000, 8, 402-410. [CrossRef]

30. Papo, N.; Shai, Y. A molecular mechanism for lipopolysaccharide protection of Gram-negative bacteria from antimicrobial peptides. J. Biol. Chem. 2005, 280, 10378-10387. [CrossRef]

31. Mireles, J.R.; Toguchi, A.; Harshey, R.M. Salmonella enterica serovar typhimurium swarming mutants with altered biofilm-forming abilities: Surfactin inhibits biofilm formation. J. Bacteriol. 2001, 183, 5848-5854. [CrossRef] [PubMed]

32. Nakao, R.; Ramstedt, M.; Wai, S.N.; Uhlin, B.E. Enhanced biofilm formation by Escherichia coli LPS mutants defective in Hep biosynthesis. PLoS ONE 2012, 7, e51241. [CrossRef] [PubMed]

33. Al-Hendy, A.; Toivanen, P.; Skurnik, M. Lipopolysaccharide O side chain of Yersinia enterocolitica O:3 is an essential virulence factor in an orally infected murine model. Infect. Immun. 1992, 60, 870-875. [CrossRef] [PubMed]

34. Meng, J.; Bai, J.Q.; Xu, J.H.; Huang, C.; Chen, J.Y. Differential regulation of physiological activities by RcsB and OmpR in Yersinia enterocolitica. FEMS Microbiol. Lett. 2019, 336, fnz210. [CrossRef] [PubMed]

35. Fang, N.; Yang, H.Y.; Fang, H.H.; Liu, L.; Zhang, Y.Q.; Wang, L.; Han, Y.P.; Zhou, D.S.; Yang, R.F. RcsAB is a major repressor of Yersinia biofilm development through directly acting on $h m s C D E, h m s T$, and hmsHFRS. Sci. Rep. 2015, 5, 9566. [CrossRef]

36. Nikaido, H. Molecular basis of bacterial outer membrane permeability revisited. Microbiol. Mol. Biol. Rev. 2003, 67, 593-656. [CrossRef]

37. Yan, F.L.; Dang, Q.F.; Liu, C.S.; Yan, J.Q.; Wang, T.; Fan, B.; Cha, D.S.; Li, X.L.; Liang, S.G.; Zhang, Z.Z. 3,6-O-[N-(2-Aminoethyl)-acetamide-yl]-chitosan exerts antibacterial activity by a membrane damage mechanism. Carbohydr. Polym. 2016, 149, 102-111. [CrossRef]

38. Matatkova, O.; Pospisilova, D.; Michailidu, J.; Jaros, P.; Masak, J. Effect of subinhibitory concentration of antibiotics on Rhodococcus erythropolis and Pseudomonas fluorescens biofilm formation. Chem. Pap. 2019, 73, 1113-1119. [CrossRef]

39. Raetz, C.R.; Whitfield, C. Lipopolysaccharide endotoxins. Annu. Rev. Biochem. 2002, 71, 635-700. [CrossRef]

40. Nagy, G.; Dobrindt, U.; Schneider, G.; Khan, A.S.; Hacker, J.; Emody, L. Loss of regulatory protein RfaH attenuates virulence of uropathogenic Escherichia coli. Infect. Immun. 2002, 70, 4406-4413. [CrossRef]

41. Bengoechea, J.A.; Skurnik, M. Temperature-regulated efflux pump/potassium antiporter system mediates resistance to cationic antimicrobial peptides in Yersinia. Mol. Microbiol. 2000, 37, 67-80. [CrossRef] [PubMed]

42. Sledjeski, D.D.; Gottesman, S. Osmotic shock induction of capsule synthesis in Escherichia coli K-12. J. Bacteriol. 1996, 178, 1204-1206. [CrossRef] [PubMed]

43. Callewaert, L.; Vanoirbeek, K.G.A.; Lurquin, I.; Michiels, C.W.; Aertsen, A. The Rcs system two-component system regulates expression of lysozyme inhibitors and is induced by exposure to lysozyme. J. Bacteriol. 2009, 191, 1979-1981. [CrossRef] [PubMed] 
44. Farizano, J.V.; Torres, M.A.; Pescaretti, M.D.L.M.; Delgado, M.A. The RcsCDB regulatory system plays a crucial role in the protection of Salmonella enterica serovar Typhimurium against oxidative stress. Microbiology 2014, 160, 2190-2199. [CrossRef]

45. Hirakawa, H.; Nishino, K.; Yamada, J.; Hirata, T.; Yamaguchi, A. Betalactam resistance modulated by the overexpression of response regulators of two-component signal transduction systems in Escherichia coli. J. Antimicrob. Chemother. 2003, 52, 576-582. [CrossRef]

46. Farris, C.; Sanowar, S.; Bader, M.W.; Pfuetzner, R.; Miller, S.I. Antimicrobial peptides activate the Rcs regulon through the outer membrane lipoprotein RcsF. J. Bacteriol. 2010, 192, 4894-4903. [CrossRef]

47. Bengoechea, J.A.; Najdenski, H.; Skurnik, M. Lipopolysaccharide O antigen status of Yersinia enterocolitica O:8 is essential for virulence and absence of $\mathrm{O}$ antigen affects the expression of other Yersinia virulence factors. Mol. Microbiol. 2004, 52, 451-469. [CrossRef]

48. Bengoechea, J.A.; Zhang, L.; Toivanen, P.; Skurnik, M. Regulatory network of lipopolysaccharide O-antigen biosynthesis in Yersinia enterocolitica includes cell envelope-dependent signals. Mol. Microbiol. 2002, 44, 1045-1062. [CrossRef]

49. Faille, C.; Lemy, C.; Allion-Maurer, A.; Zoueshtiagh, F. Evaluation of the hydrophobic properties of latex microspheres and Bacillus spores. Influence of the particle size on the data obtained by the MATH method (microbial adhesion to hydrocarbons). Colloid Surf. B Biointerfaces 2019, 182, 110398. [CrossRef]

50. Meng, J.; Xu, J.G.; Chen, J.Y. The role of osmoregulated periplasmic glucans in the biofilm antibiotic resistance of Yersinia enterocolitica. Microb. Pathog. 2020, 147, 104284. [CrossRef]

51. Meng, J.; Wang, B.Y.; Liu, D.Y.; Tao, C.; Wang, Z.W.; Zhao, X.M. High-yield anaerobic succinate production by strategically regulating multiple metabolic pathways based on stoichiometric maximum in Escherichia coli. Microb. Cell Fact. 2016, 15, 141. [CrossRef] [PubMed]

Sample Availability: Samples of the compounds are available from the authors.

Publisher's Note: MDPI stays neutral with regard to jurisdictional claims in published maps and institutional affiliations.

(C) 2020 by the authors. Licensee MDPI, Basel, Switzerland. This article is an open access article distributed under the terms and conditions of the Creative Commons Attribution (CC BY) license (http://creativecommons.org/licenses/by/4.0/). 